\title{
Mosca-dos-chifres, Haematobia irritans: comportamento e danos em vacas e bezerros Nelore antes da desmama ${ }^{1}$
}

\author{
Ivo Bianchin ${ }^{2}$ e Rafael G. O. Alves ${ }^{2}$
}

\begin{abstract}
Bianchin I. \& Alves R.G.O. 2002. [The hornfly, Haematobia irritans: behaviour and damage caused in Nellore cows and pre-weaning calves.] Mosca-dos-chifres, Haematobia irritans: comportamento e danos em vacas e bezerros Nelore antes da desmama. Pesquisa Veterinária Brasileira 22(3):109-113. Embrapa Gado de Corte, Rodovia BR 262 Km 4, Cx. Postal 154, Campo Grande, MS 79002-970, Brazil.

The hornfly and losses caused by it in Nellore cows and pre-weaning calves where studied, using two groups of 60 animals each, one group with a natural infestation and the other without hornfly, during four consecutive years. It was seen that the number of flies on the cows did not exceed an average of 80 flies/animal in all years of observation. The majority of the cows $(83 \%)$ had few flies, whereas $17 \%$ had larger numbers. The calves were infested with few flies. Rainfall in excess of $100 \mathrm{~mm}$ during short periods diminished fly infestation. Weight gain in the cows without flies (treated with insecticides) as well as their calves was greater than in the control cows, but not statistically significant $(\mathrm{P}>0.05)$. Mean percentage pregnancy during the four years was $15 \%$ more in treated cows than the non-treated animals, and this difference was probably due to the infestation of the bulls.
\end{abstract}

INDEX TERMS: Hornfly, Haematobia irritans, cattle, epidemiology, control, weight gains.

RESUMO.- Estudaram-se o comportamentos e os danos causados pela mosca-dos-chifres em vacas e bezerros Nelore antes da desmama. Foram utilizados dois grupos de 60 animais cada um, sendo um grupo com infestação natural de moscas e o outro sem moscas, por quatro anos consecutivos. Observou-se que o número de moscas das vacas não ultrapassou a média de 80 moscas/animal, em todos os anos experimentais. A maioria das vacas (83\%) apresentou poucas moscas, enquanto a minoria (17\%) teve maior quantidade. Os bezerros foram pouco infestados pelas moscas. Chuvas acima de $100 \mathrm{~mm}$ em curto espaço de tempo diminuíram a infestação de moscas. $\mathrm{O}$ ganho de peso das vacas tratadas com inseticida (sem infestação por moscas) bem como os seus bezerros foi maior do que as vacas controle, porém não foi estatisticamente significativo $(P>0,05)$. A percentagem média de prenhez, nos quatro anos, das vacas tratadas foi $15 \%$ superior a das não tratadas e esta diferença foi causada provavelmente pela infestação de moscas nos touros.

TERMOS DE INDEXAÇÃO: Mosca-dos-chifres, Haematobia irritans, bovinos, epidemiologia, controle, ganho de peso.

Classicação CNPq: 5.05.02.00-0 Medicina Veterinária Preventiva

\footnotetext{
${ }^{1}$ Aceito para publicação em 5 de agosto de 2002.

${ }^{2}$ Embrapa Gado de Corte, Rodovia BR $262 \mathrm{Km} \mathrm{4,} \mathrm{Cx.} \mathrm{Postal} \mathrm{154,} \mathrm{Campo}$ Grande, MS 79002-970, Brasil. E-mail: bianchin@cnpgc.embrapa.br
}

\section{INTRODUÇÃO}

A mosca-dos-chifres (Haematobia irritans) é um pequeno díptero $(3-5 \mathrm{~mm})$ hematófago e que parasita o hospedeiro dia e noite, abandonando-o apenas, momentaneamente, para fazer a oviposição. Quando o bovino defeca, as fêmeas voam rapidamente e depositam seus ovos em grupos de 10 a 20 , embaixo da borda da massa fecal (Honer et al. 1993). As fêmeas só colocam os ovos até 10 a15 minutos após o animal ter defecado, passado este período as fezes perdem atratividade para a mosca-dos-chifres.

Este inseto se concentra nas partes do animal que ficam fora do alcance do movimento da cabeça e cauda do animal (cupim, costas, barriga e pernas). A mosca-dos-chifres exibe preferência para bovinos de raças européias, mestiços e animais de pelagem escura ou com manchas escuras e para machos inteiros; nestes últimos a preferência está relacionada ao tamanho e maior atividade das glândula sebáceas, bem como a concentração de testosterona (Christensen \& Dobson 1979). Essa mosca pode atacar também bubalinos, equídeos e animais silvestres como o cervo, raramente ataca o cão, o ovino e o homem.

A mosca-dos-chifres é considerada uma praga em vários países. Os prejuízos desta estão relacionados a transmissão de patógenos e, principalmente, ao estresse que causa ao animal que, na tentativa de se livrar das moscas se debate muito, gastando energia, diminuindo o tempo de pastejo e ingestão de 
água. No Brasil este inseto foi identificado, pela primeira vez, em 1983, por Valério \& Guimarães (1983). Entretanto, há relatos da sua presença, em Roraima, desde 1976, tendo aparecido na maioria dos estados brasileiros em 1991 e hoje encontra-se em todo o território nacional e Países da América do Sul.

Para se estabelecer a real importância desse agente e os métodos de combatê-lo, devem-se ter dados sobre sua epidemiologia e danos causados por ele, em diversas regiões do País (Bianchin 1996). Assim, faz-se necessário o estudo da epidemiologia e dos danos que esta mosca causa nas diferentes regiões fisiográficas do Brasil.

\section{MATERIAL E MÉTODOS}

O trabalho foi desenvolvido na área experimental do Centro Nacional de Pesquisa de Gado de Corte da Empresa Brasileira de Pesquisa Agropecuária (Embrapa Gado de Corte), Campo Grande, MS, localizado a $20^{\circ} 27^{\prime} \mathrm{S}$ e $54^{\circ} 37^{\prime} \mathrm{W}$ e altitude aproximada de 530 metros, que segundo a classificação de Koëppen (Ometto 1981), situase na faixa de transição entre o clima Cfa mesotérmico úmido sem estiagem, e o clima AW tropical úmido com estação chuvosa no verão e seca no inverno. A temperatura média do mês mais quente é superior a $22^{\circ} \mathrm{C}$, apresentando no mês mais seco mais de $30 \mathrm{~mm}$ de precipitação.

O experimento foi realizado em quatro anos consecutivos: ano 1 (nov./1991 a abr./1992), ano 2 (dez./1992 a jun./1993), ano 3 (nov./ 1993 a maio/1994) e ano 4 (nov./1994 a maio/1995). A cada ano, um rebanho de 120 vacas Nelore, com bezerro ao pé nascidos entre agosto a setembro, foi dividido em dois grupos de 60 animais, levando-se em consideração as idades e os pesos das vacas e as idades dos bezerros. Durante a estação de monta (novembro a fevereiro), foram mantidos três touros, testados andrologicamente, em cada grupo. Em um dos grupos aplicaram-se, nas vacas e touros, inseticidas a base de piretróides, com intervalos que variavam de 14 a 30 dias, de modo a manter os animais livres de moscas. No outro grupo, denominado controle, os animais não receberam nenhum produto para combater a mosca. Os dois grupos foram mantidos em piquetes separados, formados de Brachiaria decumbens, rotacionados a cada 14 dias, com uma taxa de lotação de $1 \mathrm{UA} / \mathrm{há}$ ( $\pm 450 \mathrm{~kg} /$ peso/vivo).

As contagens da mosca nas vacas foram realizadas, também, a cada 14 dias. Para as contagens de moscas cada animal foi imobilizado no tronco, contando-se o número de moscas de um lado do animal e multiplicado-se o valor obtido por dois. $\mathrm{O}$ animal com o menor número de moscas por grupo, bem como aquele com número médio de infestação e aquele com maior número de moscas, no início do experimento, foram analisados durante todo o período experimental afim de se conhecer a preferência das moscas por determinado animal ("sensibilidade"). As vacas foram pesadas a cada dois meses e os bezerros somente na desmama (seis a nove meses de idade). Os animais tinham acesso, ad libitum a água proveniente de poço semi-artesiano distribuída em bebedouros de concreto e uma mistura mineral completa. As práticas de manejo animal e sanitário eram iguais para os dois grupos.

Os dados de ganho de peso das vacas e bezerros foram analisados através da análise de variância (ANOVA) utilizando-se o procedimento SAS (1989).

\section{RESULTADOS E DISCUSSÃO}

Observou-se que o uso de inseticida nas vacas do grupo tratado manteve os animais livres da mosca-dos-chifres durante todo o período experimental. A infestação nas vacas do gru- po controle, sem o uso de inseticida, não ultrapassou a média de 80 moscas/animal, em qualquer das contagens feitas durante os quatro anos experimentais. As médias anuais de infestação encontradas foram 44, 20, 31 e 24 moscas/vaca, respectivamente, para o primeiro, segundo, terceiro e quarto ano. O nível de infestação foi semelhante aos obtidos por Barros (2001) no Pantanal do Estado de Mato Grosso do Sul.

Os bezerros das vacas controle também eram observados quanto a presença de moscas, no entanto por se tratar de uma categoria animal difícil de se manejar os insetos não foram contados. As observações revelaram que os bezerros possuíam poucas ou nenhuma mosca, indicando que a mosca prefere os animais adultos. Este resultado é semelhante ao que foi constatado por Cordovés et al. 1998 e Suarez et al. 1995. As maiores infestações pela moscas nos touros em relação às vacas e bezerros está relacionada a testosterona (Dobson et al.1970).

$O$ período experimental correspondeu às épocas chuvosas quando as condições climáticas são mais propícias para o desenvolvimento da mosca-dos-chifres. A infestação observada, no presente trabalho foi baixa quando e levou em consideração os valores verificados em outros países (Steelman et al. 1991, Morrison \& Foil 1995, Suarez et al. 1995). Três fatores, no entanto, poderiam explicar essa diferença: i) raças bovinas envolvidas nos diferentes experimentos; ii) presença de inimigos naturais, tais como o besouro africano Digitonthophagus gazella, o qual foi introduzido na região há cerca de 12 anos (Bianchin et al. 1992); e iii) quantidade acumulada e ou diária de chuva que antecede cada contagem da mosca (Bianchin et al. 1993, Bianchin et al. 1995, Cordovés et al. 1998).

Apesar de o período experimental corresponder à época chuvosa, as contagens da mosca-dos-chifres foram maiores quando a soma de precipitação (14 dias antes da contagem) foi menor (Fig. 1). Uma possível explicação para este fenômeno é que, quando chove muito em poucos dias (próximo de100 $\mathrm{mm}$ ou mais), ocorre destruição das massas fecais na pastagem, interrompendo o ciclo biológico da mosca. Isto acontece provavelmente porque a mosca deposita seus ovos nas fezes recém postas que são facilmente fragmentadas e espa-

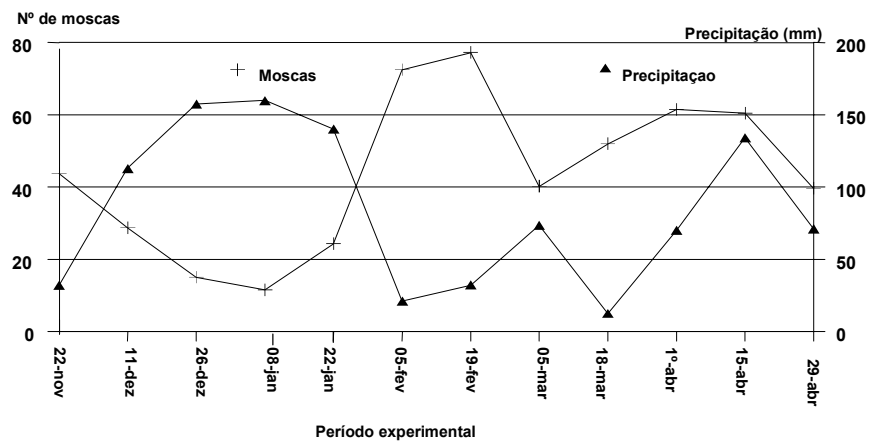

Fig. 1. Número médio de mosca-dos-chifres em vacas Nelore não protegidas por inseticidas e precipitação $(\mathrm{mm})$ acumulada entre as contagens, efetuadas a cada 14 dias, durante o período experimental de nov./1991 a abr./1992 (158 dias). 


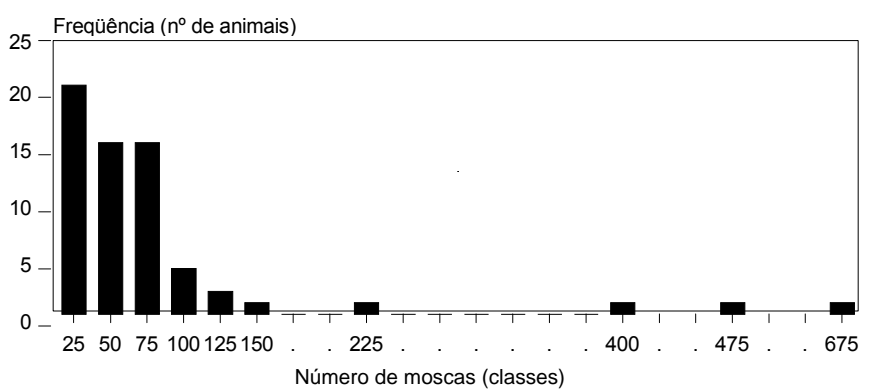

Fig. 2. Distribuição das freqüências do número de mosca-dos-chifres em 60 vacas Nelore sem o uso de inseticida na contagem de $1^{\circ}$ de abril de 1992.

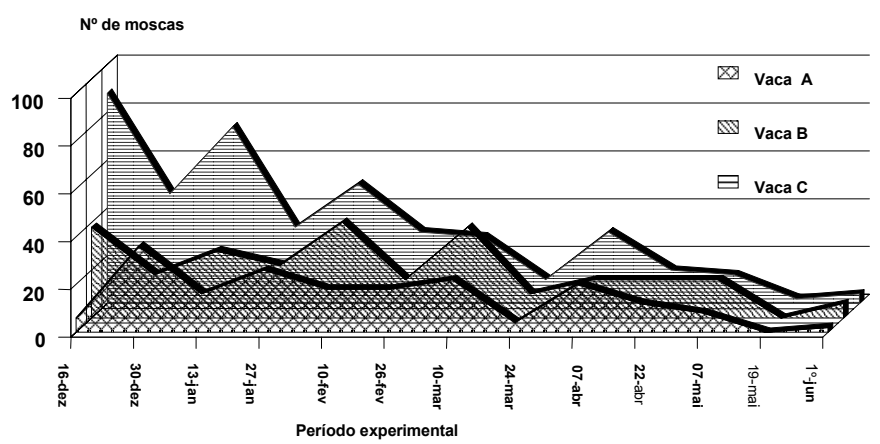

Fig. 3. Persistência de níveis de infestação da mosca-dos-chifres em vacas Nelore com 6 a 7 anos de idade. Animais que, no início do período experimental (16/dez.), tinham as seguintes infestações: menor (A), média (B) e maior (C).

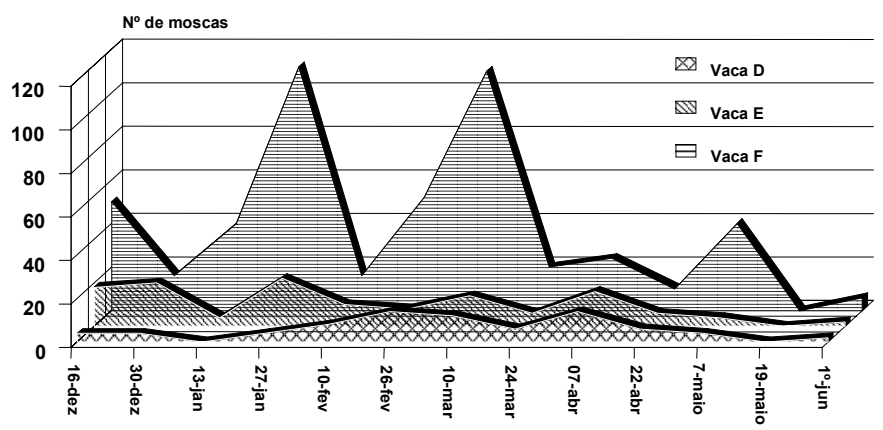

Fig. 4. Persistência de níveis de infestação da mosca-dos-chifres em vacas Nelore com 4 a 5 anos de idade. Vacas que, no início do período experimental (16/dez.), tinham as seguintes infestações: menor (D), média (E) e maior (F).

lhadas pela pastagem pela ação da chuva, e com isso destrói o ambiente favorável ao desenvolvimento e sobrevivência deste inseto.

Constatou-se, também, que cerca de $83 \%$ das vacas do grupo controle, apresentava poucas moscas, enquanto $17 \%$ tinha maior quantidade. Na contagem de $1^{\circ}$ de abril de 1992 (Fig. 2), por exemplo, de 60 vacas, 50 tinham até 75 moscas, seis tinham entre 100 e 150 e as quatro vacas restantes tinham 225, 400, 475 e 675 moscas, respectivamente.

$\mathrm{O}$ nível de infestação de mosca-dos-chifres é influenciado pela raça e coloração da pelagem e dentro da mesma raça cada animal apresenta diferentes susceptibilidades para a mosca. Assim, vacas com maior número de moscas, no início do estudo, permaneceram com maior infestação durante todo o período experimental. Relações semelhantes foram observadas para números intermediários e baixos, como por exemplo no primeiro (Fig. 3) e no segundo ano experimental (Fig. 4). Com isso, podemos dizer que existe uma variação individual podendo-se classificar os animais em resistes e não resistentes e utilizar essa informação no manejo dos animais para diminuir a infestação das moscas e o uso de inseticidas. Esses dados são semelhantes ao encontrados por Steelman et al. (1993) em algumas raças de animais de corte e alertanos sobre a possibilidade dos fator(es) responsáveis pela resistência serem hereditários, deste modo, os animais resistentes devem ser levados em consideração na seleção.

Observa-se no Quadro 1, uma tendência para as vacas tratadas com inseticidas, bem como suas crias, ganharem mais

Quadro 1. Ganho médio de peso ${ }^{\mathrm{a}}(\mathrm{kg})$ e percentagem $(\%)$ de prenhez nas vacas com (CI) e sem (SI) inseticida, e os pesos médios ao desmame (6-9 meses) dos bezerros nesses grupos. Ano 1(nov./ 1991 a abr./1992), ano 2 (dez./1992 a jun./1993), ano 3 (nov./1993 a maio/1994) e ano 4 (nov./1994 a maio/1995)

\begin{tabular}{|c|c|c|c|c|c|}
\hline \multirow[t]{3}{*}{ Ano } & \multirow{3}{*}{$\begin{array}{r}\text { Grupo } \\
(\mathrm{n}=60)\end{array}$} & \multicolumn{3}{|c|}{ Vacas } & \multirow{3}{*}{$\begin{array}{c}\text { Bezerro } \\
(\mathrm{kg})\end{array}$} \\
\hline & & \multirow{2}{*}{$\begin{array}{c}\text { Ganho } \\
\text { (kg) }\end{array}$} & \multicolumn{2}{|c|}{ Prenhez (\%) } & \\
\hline & & & Lote & Diferença & \\
\hline \multirow[t]{2}{*}{1} & $\mathrm{Cl}$ & $74^{\mathrm{b}}$ & 63 & 4,8 & $168^{b}$ \\
\hline & $\mathrm{SI}$ & $67^{b}$ & 60 & & $165^{\mathrm{b}}$ \\
\hline \multirow[t]{2}{*}{2} & $\mathrm{Cl}$ & $8^{\mathrm{b}}$ & 83 & 15,7 & $135^{\mathrm{b}}$ \\
\hline & SI & $7^{b}$ & 70 & & $133^{b}$ \\
\hline \multirow[t]{2}{*}{3} & $\mathrm{CI}$ & $16^{\mathrm{b}}$ & 54 & 25,9 & $125^{\mathrm{b}}$ \\
\hline & SI & $14^{\mathrm{b}}$ & 40 & & $124^{\mathrm{b}}$ \\
\hline \multirow[t]{2}{*}{4} & $\mathrm{Cl}$ & $39^{b}$ & 83 & 12 & $152^{b}$ \\
\hline & SI & $36^{\mathrm{b}}$ & 73 & & $150^{\mathrm{b}}$ \\
\hline
\end{tabular}

avalores na mesma coluna e dentro do ano com a mesma letra não são significativamente diferentes $(\mathrm{P}>0,05)$.

peso do que os animais do grupo controle, embora essas diferenças não tenham sido estatisticamente significativas $(\mathrm{P}>0,05)$. As diferenças de ganhos de peso médio foram de $7,1,2$ e $3 \mathrm{~kg}$ para as vacas e de 3, 2, 1 e $2 \mathrm{~kg}$ para os bezerros à desmama, no primeiro, segundo, terceiro e quarto ano, respectivamente. Estes resultados indicam que o nível de infestação da mosca produziu pouco efeito no ganho de peso de vacas e bezerros Nelore antes da desmama e são similares aos obtidos por outros estudos (Schreiber et al. 1987, Hogsette et al. 1991, Morrison \& Foil 1995). Os resultados diferem dos estudos realizados por Kunz et al. (1984), Quisenberry \& Strohbehn (1984), Haufe (1986), Steelman et al. (1991) e Suarez et al. 1995, que encontraram diferenças significativas no ganho de peso de vacas e bezerros tratados contra a mosca-dos-chifres. Os contrastes de resultados observados entre anos em uma mesma região ou regiões diferentes, se devem ao grau de infestação dos animais, resultan- 
tes das condições climáticas, manejo, raça e alimentação próprios de cada local.

O uso de inseticida aumentou em relação ao grupo não tratado $4,8 \%, 15,7 \%, 25,9 \%$ e $12 \%$ a percentagem de prenhez , respectivamente, para o primeiro, segundo, terceiro e quarto ano.

Levando-se em consideração a baixa infestação por moscas e a inexistência do efeito de tratamento no ganho de peso das vacas e bezerros, supõe-se que a diferença no índice de prenhez seja devida ao estresse dos touros que quase sempre apresentaram altas infestações pela mosca-dos-chifres (acima de 500). Com isso, sugere-se controlar a mosca-dos-chifres nos touros, durante a estação de monta, podendo neste caso se utilizar brincos com maior poder residual. Nas vacas, levando-se em consideração que poucas possuem grande quantidades de moscas e são sempre os mesmos animais, estas seriam tratadas conforme recomendação de Cordovés et al. (1998) que obteve um controle por cerca de 40 dias tratando $27 \%$ das vacas mais infestadas. Desta forma estaria se reduzindo o uso de produtos químicos e consequentemente diminuindo o risco de resistência e danos ao ambiente, preservando o controle biológico especialmente os besouros e havendo, portando, uma integração entre o controle químico e o biológico.

$\mathrm{O}$ besouro africano foi introduzido praticamente em todos os estados do Brasil e tal feito se deve à colaboração dos órgãos de pesquisa (federais e estaduais), sindicatos rurais e da iniciativa privada. Em Mato Grosso do Sul este besouro encontra-se amplamente estabelecido, com exceção das áreas inundáveis do Pantanal, no qual as condições ambientais parecem ser desfavoráveis ao seu desenvolvimento.

0 besouro tem mostrado boa adaptabilidade às condições brasileiras e aparece em maior número durante o período chuvoso e quente. Já foram realizados no País alguns estudos sobre a importância do $D$. gazella na destruição de bolos fecais e as suas consequiências para o solo e plantas (Galbiati et al. 1995a,b, Miranda et al. 1998, 2000).

Fica evidente, portanto, a necessidade de estudos, em diferentes regiões do País, para que se possam conhecer a epidemiologia e os danos que a mosca-dos-chifres causa e, com isso, se propor métodos de controles integrados que sejam eficientes e econômicos. Os inimigos naturais podem limitar a reprodução do inseto nas fezes em cerca de $90 \%$ e acredita-se que, sem eles o número de moscas poderia ser insuportavelmente elevado (Honer et al. 1993). Salienta-se ainda que, a quase totalidade dos inseticidas existentes no mercado age nas fezes bovinas, eliminando, por exemplo, os besouros (Bianchin et al. 1997, 1998). Portanto, deve-se usar o mínimo de inseticida nos animais afim de minimizar a contaminação da carne e do leite e preservar o ambiente.

Agradecimentos.- Os autores agradecem ao Laboratorista Ananias Loveira e ao Técnico Agrícola Paulino Gauna Gomes pelo auxílio nos trabalhos de laboratório e campo.

\section{REFERÊNCIAS}

Barros A.T.M. 2001. Dynamics of Haematobia Irritans irritans (Diptera:Muscidae) infestation on Nelore cattle in the Pantanal, Brasil. Mem. Inst. Oswaldo Cruz 94(4):445-450.
Bianchin I. 1996. Mosca-dos-chifres: comportamento e danos em bovinos Nelore. I ${ }^{\circ}$ Simpósio sobre Controle de Parasitos. Programa e palestras: controle de parasitos em ruminantes. Campinas-SP, p.46-49.

Bianchin I., Honer, M.R. \& Gomes A. 1992. Controle integrado da mosca-dos-chifres na Região Centro-Oeste. Hora Vet. 65:43-46.

Bianchin I., Honer, M.R., Koller W.W., Gomes A. \& Schenk J.A.P. 1993. Dinâmica populacional e efeito da mosca-dos-chifres (Haematobia irritans) sobre vacas e bezerros nelore. $8^{\circ}$ Semin. Bras. Parasitol. Vet., Londrina, PR., p. 30. (Resumo)

Bianchin I., Honer M.R., Dode M.A.N., Koller W.W., Gomes A. \& Vaz, E.C. 1995. Population dynamics and effect of the hornfly (Haematobia irritans) on Nellore cows and calves. IX Semin. Bras. Parasitol. Vet., Campo Grande, MS, p.a13. (Resumo)

Bianchin I., Alves R.G.O. \& Koller W.W. 1997. Efeito de carrapaticidas/inseticidas aspersão sobre adultos do besouro coprófago africano Onthophagus gazella (F.). Ecossistema 22:116-119.

Bianchin I., Alves R.G.O. \& Koller W.W. 1998. Efeito de carrapaticidas/inseticidas "pour-on" sobre adultos do besouro coprófago africano Onthophagus gazella Fabr. (Coleoptera: Scarabaeidae). Anais Soc. Entomol. Bras. 27(2):275279.

Cordovés C.O., Souza M.O.L., Lorenzoni H., Pontes, J.B. \& Baltodano, R.J.P. 1998. Epizootiologia e eficácia da cipermetrina 5\%, formulação “poor on”, em infestações naturais de Haematobia irritans. Hora Vet. 105:77-81.

Christensen C.M. \& Dobson R.C. 1979. Effects of testosterone propionate on the sebaceous glands and subsequent attrativeness of Angus bulls and steers to horn flies, Haematobia irritans (Diptera: Muscidae). J. Kans. Entomol. Soc. 52:386-391.

Dobson, R.C., Kutz F.W. \& Sanders, D.P. 1970. Attraction of horn flies to testosterone-treated steers. J. Econ. Entomol. 63(1):323-324.

Galbiati C., Bensi C., Conceição C.H.C., Florcovski J.F. \& Calafiori M.H. 1995a. Estudo comparativo entre besouros do esterco Dichotomius anaglypticus (Mann.,1829) e Onthophagus gazella (F.), sobre as pastagens, em condições brasileiras. Ecossistema 20:109-118.

Galbiati C., Conceição C.H.C., Florcovski J.F., Calafiori M.H. \& Tobias, A.C.T. 1995b. Efeito de vermífugos injetáveis em bovinos de leite sobre o besouro coprófago Dichotomius anaglypticus (Mann.1829). Ecossistema 20:100-108.

Haufe W.O. 1986. Productivity of the cow-calf unit in range cattle protected from horn flies, Haematobia irritans (L.), by pesticidal ear tags. Can. J. Anim. Sci. 66:575-589.

Hogsette J.A., Prichard D.L. \& Ruff J.P. 1991. Economic effects of horn fly (Diptera: Muscidae) populations on beef cattle exposed to three pesticide treatment regimes. J. Econ. Entomol. 84:1270-1274.

Honer M.R., Bianchin I. \& Gomes A. 1993. Mosca-dos-chifres: histórico, biologia e controle. Documentos 45, Embrapa-CNPGC, Campo Grande. 34 p.

Kunz S.E., Allen J.A., Sims P.L. \& Meyerhoeffer D.C. 1984. Economics of controlling horn flies (Diptera: Muscidae) in range cattle. J. Econ. Entomol. 77:657-660.

Miranda C.H.B., Santos J.C.C. \& Bianchin I. 1998. Contribuição de Onthophagus gazella à melhoria da fertilidade do solo pelo enterrio de massa fecal bovina fresca. I. Estudo em casa de vegetação. Revta Bras. Zootec. 27(4):681685.

Miranda C.H.B., Santos J.C.C. \& Bianchin I. 2000. The role of Digitonthophagus gazella in pasture cleaning and production as a result of burial of cattle dung. Pasturas Trop. 22(1):14-18.

Morrison D.G. \& Foil L.D. 1995. Effect of horn fly (Diptera:Muscidae) control during the spring on calf production by fall-calving beef cows. Vet. Entomol. 88(1):81-84.

Ometto J.C. 1981. Bioclimatologia vegetal. Ed. Agronômica Ceres, São Paulo. 425 p.

Quisenberry S.S. \& Strohbehn D.R. 1984. Horn fly (Diptera: Muscidae) control of beef cows with permethrin-impregnated ear tags and effect on subsequent calf weight gains. J. Econ. Entomol. 77:422-424. 
SAS Institute Inc. 1989. SAS/Stat User's Guide, Version 6. Vol. 1. $4^{\text {th }}$ ed. SAS Institute, Cary, NC. 943 p.

Schreiber E.T., Campbell J.B. Kunz S.E., Clanton D.C. \& Hudson D.B. 1987. Effects of horn fly (Diptera: Muscidae) control on cows and gastrointestinal worm (Nematode: Trichostrongylidae) treatment for calves on cow and calf weight gains. J. Econ. Entomol. 80:451-454.

Steelman C.D., Brown Jr. A.D., Gbur, E.E. \& Tolley G. 1991. Interactive response of the horn fly (Diptera: Muscidae) and selected breeds of beef cattle. J. Econ. Entomol. 84 (4):1275-1282.
Steelman C.D., Gbur E.E., Tolley G. \& Brown Jr A.D. 1993. Individual variation within breeds of beef cattle in resistence to horn fly (Diptera:Muscidae). J. Med. Entomol. 30 (2):414-420.

Suarez V.H., Fort M.C. \& Busetti M.R. 1995. Observaciones del efecto de la mosca de los cuernos en al comportamiento y la productividad de la cria bovina en la region semiarida pampeana. Revta Med. Vet. 76(2):83-87.

Valério J.R. \& Guimarães J.H. 1983. Sobre a ocorrência de uma nova praga, Haematobia irritans (L.) (Diptera: Muscidae), no Brasil. Revta Bras. Zool. 1(4):417-418. 